\title{
Evaluation of Diagnostic Values in NCCT and MRI of the Patients With Cerebral Venous or Sinus Thrombosis in Loghman Hakim Hospital in Tehran 2014-2018
}

\author{
Hossein Hatami ${ }^{1}$, Niyousha Danesh ${ }^{2 *}$, Maziar Shojaei ${ }^{3}$, Amir Rabie Hamedani ${ }^{4}$ \\ 1Department of Public Health, School of Public Health, Shahid Beheshti University of Medical Science, Tehran, Iran \\ 2Department of MPH, School of Public Health, Shahid Beheshti University of Medical Sciences, Tehran, Iran \\ ${ }^{3}$ Department of Neurology, Loghman Hakim Hospital, Shahid Beheshti University of Medical Sciences, Tehran, Iran \\ ${ }^{4} \mathrm{MSchool}$ of Medicine, Shahid Beheshti University of Medical Sciences, Tehran, Iran
}

\begin{abstract}
Background: Cerebral venous and sinus thrombosis (CVT) is the rare type of stroke, which can be fatal or causes significant morbidity. CVT could cause by multiple etiologies and risk factors and could present with many signs and symptoms such as a headache, seizure or altered mental status. The gold standard for diagnosing CVT is magnetic resonance venography (MRV) or computed tomographic venography (CTV), as these modalities are not available in many health centers and the early diagnosis of this disease improves significantly the outcome of treatment, we evaluated the diagnostic values of CT and magnetic resonance imaging (MRI) in CVT patients.

Methods: We compiled and investigated the radiological and clinical records of 92 patients with the final diagnosis of CVT; we observed the sign of thrombosis in 74 patients who had either the non-contrast CT or MRI plus MRV. We statistically analyzed the sensitivity, specificity, positive predictive value, negative predictive value, accuracy, the positive and negative likelihood ratio of these modalities.

Results: Our results demonstrated that the sensitivity of CT scan is $60.5 \%$, and its specificity is $66.7 \%$. The positive predictive value of CT is $85 \%$, and its negative predictive value is $34 \%$. Our study demonstrated that the accuracy of CT in diagnosing CVT patients is $62 \%$.

Besides our results showed that the sensitivity of MRI in diagnosing CVT is $87 \%$, and the specificity of MRI is $76.9 \%$. Hence the positive predictive value of MRI is $94 \%$, and the negative predictive value is $58 \%$, and the accuracy of this modality is $85 \%$

Conclusion: In the absence of gold standard modalities, MRI and CT scan have relatively good diagnostic values, besides MRI is more beneficial between the two, moreover we found that CVT is more prevalent among women and in warm seasons.

Keywords: Cerebral venous and sinus thrombosis (CVT); CT; MRI; MRV; Diagnosis.
\end{abstract}

*Correspondence to Niyousha Danesh (MD-MPH) Department of MPH, School of Public Health, Shahid Beheshti University of Medical Sciences, Tehran, Iran.

Tel: +98-9122380334; Email: newdan8@gmail.co

Published online 20 March 2019

Citation: Hatami H, Danesh N, Shojaei M, Rabie Hamedani A. Evaluation of diagnostic values in NCCT and MRI of the patients with cerebral venous or sinus thrombosis (CSVT) in Loghman Hakim Hospital in Tehran 2014-2018. Int Clin Neurosci J. 2019;6(1):17-21. doi:10.15171/icnj.2019.04.

\section{Introduction}

Cerebral venous thrombosis (CVT) and dural sinus thrombosis are rare types of brains strokes, which has a prevalence of 5 per 1 million and include about $0.5-1 \%$ of strokes. In opposed to other types of stroke CVT is especially prevalent in young patients (less than 50 years old), and its median age is $37 .{ }^{1}$

CVT has various presentations; however, headache is the most frequent chief complaint in these patients. These patients could present with seizures, altered mental status, focal neurologic deficits, like aphasia, paresthesia, and paraplegia, intracranial hypertension, papilledema, visual disorders such as diplopia, sixth nerve palsy, and encephalopathy. ${ }^{2-4}$

Broad range of risk factors can cause CVT, including oral contraceptives, pregnancy and puerperium, ${ }^{5}$ head and neck infections, drugs like steroids, hormone therapy and chemotherapy, head trauma, thrombophilias, dysfibrinogenemia, antiphospholipid syndrome, 6 protein C, protein S or antithrombin III deficiency, increased factor VIII, prothrombin 20210A mutation, mutation of factor $\mathrm{V}$ Leiden and minor procedures. ${ }^{5,7-9}$

CVT can be fatal, and its mortality rate is about $10 \%$ to $20 \%$. Moreover, CVT's morbidity is related to the focal neurological deficit and recurrent seizures, which are the significant consequences of $\mathrm{CVT}^{1,9}$ so diagnosing 
these patient as soon as possible could improve clinical outcomes and reduce CVT's mortality and morbidity rate.

Computed tomography (CT), magnetic resonance imaging (MRI), magnetic resonance venography (MRV), CT venography (CTV) and digital subtraction angiography (DSA) remain the diagnostic neuroimaging modalities for cerebral venous or dural sinus thrombosis in patients manifesting the presentations as mentioned earlier. ${ }^{10,11}$

MRV and CVT are most accurate in thrombosis detection. ${ }^{12}$ However, these devices are not available in all clinics and hospitals, meanwhile many of these centers equipped to CT and MRI apparatus. Hence evaluation of the diagnostic values of CT and MRI could lead to correct diagnosis and accelerate the appropriate treatment which is anticoagulation and thrombolytic therapy.

This study aims to retrospectively investigate the findings of thrombosis in CT and MRI of patients with CVT in order to evaluate the diagnostic values of these neuroimaging modalities.

\section{Methods}

We used the electronic in-hospital database of Loghman Hakim hospital in this retrospective single-center hospital-based study. We compiled and investigated the radiological and clinical records of 92 patients with the final diagnosis of CVT and dural sinus thrombosis irrespective of sex and age from March 2014 until June 2018.

All patients presenting with the diagnostic criteria of CVT, confirmed using noncontrast CT (NCCT) or MRI, followed by MRV or CTV included in our study, but 18 patients who did not have radiological images and reports of either CT, MRI or CTV, MRV or with low-quality images excluded from analysis. Patients with the sole presentation of intracranial hemorrhage (ICH) and the absence of thrombosis in NCCT excluded either.

All MRV, CTV, MRI, and CT without contrast images were observed and reviewed by one neurologist and one radiologist. In 2 cases we used CTV for confirmation.

In NCCT studies we used direct signs of thrombosis in cortical vein or sinus represents the possibility of CVT. We searched for spontaneous density, cord sign and triangle sign in NCCT.

In the contrasted CT, prominent vascular or dural enhancement is a sign of thrombosis the filling defect of empty delta sign could also demonstrate the superior sagittal sinus or transverse or sinus thrombosis. We equally considered evidence of hemorrhagic infarction or ICH subarachnoid hemorrhage and cerebral hematoma as a possibility of CVT.

In MRI studies we investigated the presence of thrombus in T1-weighted images of MRI or changes in intensity and inhomogeneous signal abnormality, i.e., hypointensity on $\mathrm{T} 2$-weighted images in the acute phase ( first five days) and hyperintensity on $\mathrm{T} 1$ and T2-weighted or FLAIR images in the subacute phase.

MRV or CTV performed in all cases for confirmation of CVT diagnosis. We compared these presentations in CT and MRI with loss of signal in the sinus or venous on MRV or the visualization of thrombosis in CT venogram (CTV).

\section{Statistical Analysis}

All the acquired data were transformed into a database using Microsoft Excel, then the statistical measures, sensitivity, specificity, positive predictive value, negative predictive value, positive and negative likelihood ratio, and accuracy was calculated using SPSS 21 . The frequency of females with CVT vs. males and the CVT patients in warm seasons vs. in cold season were also measured.

\section{Results}

\section{Clinical Data}

Ninety-two patients with first and final diagnosis of cerebral venous and sinus thrombosis observed. Sixtytwo persons (67\%) were female, and 30 persons (32\%) were male. In the proportion of male to female ratio, as we expected the rate of CVT in women was more significant (Table 1).

As Table 1 shows, 61 (66\%) of CVT cases have been occurred in the hot season (spring and summer in the Northern hemisphere). Hence 31 (34\%) of CVT cases happened in the cold seasons (autumn and winter).

From 92 patients recorded with the diagnosis of CVT, 18 patients had been excluded in our imaging study, as they lacked either MRV or one of the modalities of CT or MRI, therefore the radiologic images and reports of 74 patients with CVT, 52 female (70.3\%) and 22 (29.7\%) male, were investigated.

Fifty CVT patients had both radiologic images of CT and MRV, from which four patients (8\%) demonstrated false positive and 15 patients (30\%) had false negative results.

We found that the sensitivity of CT scan in diagnosing cerebral venous/sinus thrombosis is $60.5 \%$, and the specificity of CT is $66.7 \%$. The positive predictive value of $\mathrm{CT}$ is $85 \%$, and its negative predictive value is $34 \%$. Our study demonstrated that the accuracy of CT in diagnosing

Table 1. Frequency and Percentage of CVT Among Gender and Seasonal Differences

\begin{tabular}{lcc}
\hline CVT & No. & Percent \\
\hline Male & 30 & 32 \\
Female & 62 & 67 \\
Hot season & 61 & 66 \\
Cold season & 31 & 34 \\
Total & 92 & 100 \\
\hline
\end{tabular}


CVT patients is $62 \%$ moreover the positive and negative likelihood ratio for this modality is 1.81 and 0.59 (Table 2).

Whereas 67 patients had both MRI and MRV images and reports, among them three patients with CVT (4.4\%) had a false positive result in their MRI, and seven patients $(10.4 \%)$ had false negative results.

Our results showed that the sensitivity of MRI in diagnosing cerebral venous/ sinus thrombosis is $87 \%$, and the specificity of MRI is $76.9 \%$. Hence the positive predictive value of MRI is $94 \%$, and the negative predictive value is $58 \%$. Table 2 also reveals that the accuracy of this modality in diagnosing CVT patients is $85 \%$ moreover the positive and negative likelihood ratio of MRI is 5.91 and 0.26 .

\section{Discussion}

Even though different modalities for recognition of thrombosis in cerebral venous or sinus, many patients with CVT are misdiagnosed or not diagnosed at all. CVT affects both sexes and all age groups, including many young individuals and if they go untreated, severe complication or even death could occur. Therefore, the accurate and rapid diagnosis of CVT is crucial. Clinical presentations of CVT varies in each patient and laboratory findings are not diagnostic. The standard gold test for CVT is MRV or CTV, ${ }^{13}$ but many clinics cannot operate MRV, so we evaluated the statistical values of CT scan and MRI in suspicious cases, in the case that the modalities as mentioned earlier are not available. Our study indicated that from 82 patients with cerebral venous and sinus thrombosis, 62 patients were female and 30 patients were male. In the proportion of male to female ratio, as we expected the rate of females with CVT was higher, which is compatible with previous studies and it is due to OCP usage and disorders increasing hypercoagulability in women such as antiphospholipid antibody syndrome, ${ }^{6,14-16}$ although the rate of CVT in male patients is a little higher than some previous studies. ${ }^{11,17,18}$

We found that thromboses in venous and sinuses happen more often in summer or spring than in winter and autumn. This warm season tendency is due to more body dehydration in these seasons, which increases the risk of thrombosis. The most common presentation was a headache, compatible with other studies. ${ }^{19-21}$

Our results demonstrated that the sensitivity of CT scan in the diagnosis of CVT is $60.5 \%$ and the specificity of CT is $66.7 \%$. These amounts are lower than the study by Linn et al, indicating sensitivity of $64.6 \%$ and specificity of $97.2 \%$ only for the cord sign. ${ }^{17}$ Moreover, the study by Avsenik et al showed the sensitivity of $100 \%$ and specificity of $83 \%$ in NCCT in patients with CVT. ${ }^{10}$ Probably due to our larger sample size and because we did not include the finding of intracerebral edema alone in our positive results.

Our positive results consist of intracerebral hemorrhage, attenuated vein sign or cord sign presentation in CT
Table 2. Diagnostic validity of CT and MRI in CVT

\begin{tabular}{lcc}
\hline Validity & NCCT & MRI \\
\hline Sensitivity & $60.5 \%$ & $87 \%$ \\
Specificity & $66.7 \%$ & $76.9 \%$ \\
Positive predictive value & $85 \%$ & $94 \%$ \\
Negative predictive value & $34 \%$ & $58 \%$ \\
Positive Likelihood ratio & 1.81 & 5.91 \\
Negative Likelihood ratio & 0.59 & 0.26 \\
Accuracy & $62 \%$ & $85 \%$ \\
Total patients & 50 & 67 \\
\hline
\end{tabular}
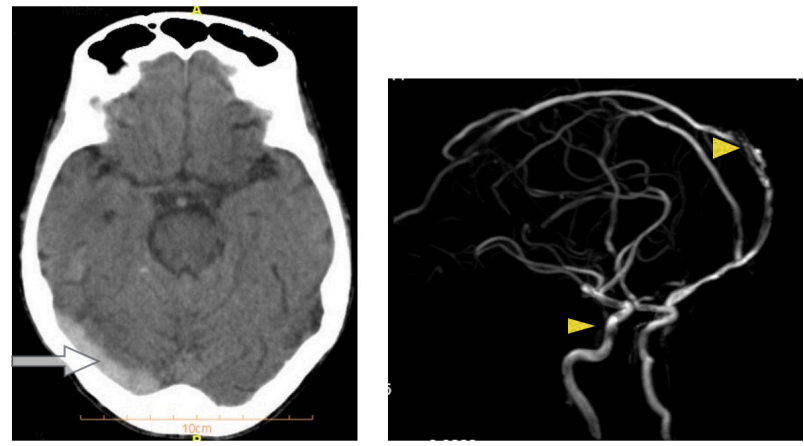

Figure 1. A 45-Year-Old Woman With CVT. (A) The Noncontrast CT (NCCT) shows the true positive cord sign in the right transverse sinus (arrow) and (B) indicates the bilateral transverse and superior sagittal sinus thrombosis in the MRV (arrowhead).

images (Figure 1A).

Our CT results indicated lower percentage of false positive and false negative compared to the study by Tang et $\mathrm{al}^{22}$ (52.6\% false negative). Four patients had false positive (8\%) and 15 patients had false negative (30\%) results. The study by Linn et $\mathrm{al}^{17}$ showed the false positive rate almost same as our results ( 5 persons), although their false negative rate was higher (28 patients).

Our study represents the more considerable value of CT scan in the diagnosis of thrombosis in CVT patients. Moreover the positive predictive value and negative predictive value of CT was $85 \%$ and $34 \%$, these results demonstrate the higher probability of the disease in the positive NCCT, however the negative result cannot rule out the CVT.

The positive likelihood ratio of 1.81 indicates the more significant benefit of CT for ruling in CVT in individuals with positive results.

Furthermore, the accuracy of CT is $62 \%$, which together with all of the other findings state that manifestation of thrombosis in CT images in the absence of MRV or CTV neither certainly approve the diagnosis of CVT nor rule it out.

CT images could use as the first step in suspicious CVT patients in the absence of other modalities, yet they must verify by MRV (Figure 1B) or CTV. 
Our findings showed that MRI has a sensitivity of $94 \%$ and specificity of $58 \%$ in diagnosing cerebral venous and sinus thrombosis. In the study by Daghighi et al, ${ }^{13}$ the specificity of MRI was also relatively low, comparing to MRI's sensitivity and MRV. Three individuals (4.4\%) had false-positive results, and seven individuals (10.4\%) had false negative results. The similar study by Tang et $\mathrm{al}^{22}$ stated $11 \%$ false negative rate for MRI, which is higher compared to our findings.

The $94 \%$ positive predictive value of MRI indicates the significance of its positive results is, although the negative predictive value is $58 \%$ so it cannot rule out the diagnosis of CVT. We found that the accuracy of this modality in diagnosing thrombosis in the sinus or venus is $85 \%$, which does not vary significantly from MRV or CTV.

The positive likelihood ratio (+LR) of MRI is 5.91 which is much higher than 1 and indicates that the positive result highly associated with CVT. Hence the negative likelihood ratio of MRI is 0.26 , which these high variations between $+\mathrm{LR}$ and the -LR amounts reveal the more significant potential of MRI in diagnosing the cerebral thrombosis.

\section{Conclusion}

Cerebral venous and sinus thrombosis could be fatal, and it has a high morbidity rate so a rapid diagnosis of this disease would decrease its mortality and morbidity rate. Although the standard gold test for diagnosing CVT is MRV or CTV, in the absence of this modality MRI and CT scan have relatively good diagnostic values, besides MRI is more beneficial between the two and the positive results of MRI suggest the thrombosis strongly in the cerebral sinus or vein. The prevalence of CVT is more prevalent among women, the probable reason is OCP, so women should be more concerned about using it. Thrombosis in venous or sinuses also occurs frequently in warm seasons rather than cold seasons. Therefore individuals with the risk factor for thrombosis should avoid becoming dehydrated in these seasons.

\section{Conflict of Interest Disclosures}

The authors declare that they have no conflict of interests.

\section{Ethical Statement}

This article was approved by Shahid Beheshti University with ethics code IR.SBMU.PHNS.REC.1397.19.

\section{Acknowledgment}

The authors thank the staff and the neurology department of Loghman Hakim hospital for the cooperation in data collection and the department of public health in Shahid Beheshti university. This study has not received any grant from a public, nonprofit or commercial institute. The authors declare that there is no conflict of interest.

\section{References}

1. Ferro JM, Canhao P. Cerebral venous sinus thrombosis: update on diagnosis and management. Curr Cardiol Rep.
2014;16(9):523. doi: 10.1007/s11886-014-0523-2.

2. deVeber G, Andrew M, Adams C, Bjornson B, Booth F, Buckley DJ, et al. Cerebral sinovenous thrombosis in children. N Engl J Med. 2001;345(6):417-23. doi: 10.1056/ nejm200108093450604.

3. Bousser MG, Ferro JM. Cerebral venous thrombosis: an update. Lancet Neurol. 2007;6(2):162-70. doi: 10.1016/ s1474-4422(07)70029-7.

4. Stam J. Thrombosis of the cerebral veins and sinuses. N Engl J Med. 2005;352(17):1791-8. doi: 10.1056/NEJMra042354.

5. Coutinho JM, Ferro JM, Canhao P, Barinagarrementeria F, Cantu C, Bousser MG, et al. Cerebral venous and sinus thrombosis in women. Stroke. 2009;40(7):2356-61. doi: 10.1161/strokeaha.108.543884.

6. Carhuapoma JR, Mitsias P, Levine SR. Cerebral venous thrombosis and anticardiolipin antibodies. Stroke. 1997;28(12):2363-9.

7. Lijfering WM, Brouwer JL, Veeger NJ, Bank I, Coppens M, Middeldorp S, et al. Selective testing for thrombophilia in patients with first venous thrombosis: results from a retrospective family cohort study on absolute thrombotic risk for currently known thrombophilic defects in 2479 relatives. Blood. 2009;113(21):5314-22. doi: 10.1182/ blood-2008-10-184879.

8. Martinelli I, Bucciarelli P, Passamonti SM, Battaglioli T, Previtali E, Mannucci PM. Long-term evaluation of the risk of recurrence after cerebral sinus-venous thrombosis. Circulation. 2010;121(25):2740-6. doi: 10.1161/ circulationaha.109.927046.

9. Bushnell C, Saposnik G. Evaluation and management of cerebral venous thrombosis. Continuum (Minneap Minn). 2014;20(2 Cerebrovascular Disease):335-51. doi: 10.1212/01.CON.0000446105.67173.a8.

10. Avsenik J, Oblak JP, Popovic KS. Non-contrast computed tomography in the diagnosis of cerebral venous sinus thrombosis. Radiol Oncol. 2016;50(3):263-8. doi: 10.1515/ raon-2016-0026.

11. Kiran Kumar A, Venkateshwarlu J, Kumar S. Role of MRI in Evaluation of Cerebral Venous Thrombosis. IOSR Journal of Dental and Medical Sciences. 2018;17(6):6-12. doi: 10.9790/0853-1706110612.

12. Daghighi $M H$, Poureisa $M$, Safarpour $M$, Behzadmehr R, Fouladi DF, Meshkini A, et al. Diffusion-weighted magnetic resonance imaging in differentiating acute infectious spondylitis from degenerative Modic type 1 change; the role of b-value, apparent diffusion coefficient, claw sign and amorphous increased signal. $\mathrm{Br} J$ Radiol. 2016;89(1066):20150152. doi: 10.1259/bjr.20150152.

13. Daghighi $\mathbf{M H}$, Golestani S, Taheraghdam A. Comparison of Diagnostic Value of MRI and MRV with MRI/MRV Combination in Cerebral Venous Thrombosis. American Journal of Life Science Researches. 2016;4(1):7-8.

14. Prochazka V, Rajner J, Prochazka M, Dvorak J, Cizek V. Oral contraceptive induced cerebral venous thrombosis treated by local catheter directed thrombolysis. Interv Neuroradiol. 2004;10(4):321-8. doi: 10.1177/159101990401000406.

15. Amoozegar F, Ronksley PE, Sauve R, Menon BK. Hormonal contraceptives and cerebral venous thrombosis risk: a systematic review and meta-analysis. Front Neurol. 2015;6:7. doi: 10.3389/fneur.2015.00007.

16. Xu F, Liu C, Huang X. Oral contraceptives caused venous sinus thrombosis complicated with cerebral artery infarction 
and secondary epileptic seizures: A case report and literature review. Medicine (Baltimore). 2017;96(51):e9383. doi: 10.1097/md.0000000000009383.

17. Linn J, Pfefferkorn T, Ivanicova K, Muller-Schunk S, Hartz $S$, Wiesmann $M$, et al. Noncontrast $C T$ in deep cerebral venous thrombosis and sinus thrombosis: comparison of its diagnostic value for both entities. AJNR Am J Neuroradiol. 2009;30(4):728-35. doi: 10.3174/ajnr.A1451.

18. Foroughipoor M, Moghaddam Ahmadi A, Fadaei S, Ravankhah Moghaddam K, Sharifi Razavi A. Causes of cerebral venous thrombosis and its clinical manifestation in patients admitted in emergency unit and neurology ward of Ghaem hospital. Journal of Mazandaran University of Medical Sciences. 2013;23(98):86-90.

19. Cumurciuc R, Crassard I, Sarov M, Valade D, Bousser MG.
Headache as the only neurological sign of cerebral venous thrombosis: a series of 17 cases. J Neurol Neurosurg Psychiatry. 2005;76(8):1084-7. doi: 10.1136/jnnp.2004.056275.

20. Wasay M, Kojan S, Dai Al, Bobustuc G, Sheikh Z. Headache in cerebral venous thrombosis: incidence, pattern and location in 200 consecutive patients. J Headache Pain. 2010;11(2):137-9. doi: 10.1007/s10194-010-0186-3.

21. Botta R, Donirpathi S, Yadav R, Kulkarni GB, Kumar MV, Nagaraja D. Headache patterns in cerebral venous sinus thrombosis. J Neurosci Rural Pract. 2017;8(Suppl 1):S72-s7. doi: 10.4103/jnrp.jnrp_339_16.

22. Tang PH, Chai J, Chan YH, Chng SM, Lim CC. Superior sagittal sinus thrombosis: subtle signs on neuroimaging. Ann Acad Med Singapore. 2008;37(5):397-401. 